\title{
DIFFERENTIAL ALGEBRAIC DESCRIPTION OF BEAM DYNAMICS TO VERY HIGH ORDERS
}

\author{
M. BERZ \\ sisc Central Design Group, Universities Research Association, Lawrence Berkeley \\ Laboratory, University of California, Berkeley, CA 94720
}

(Received March 14, 1988)

The new method of differential algebras for the description of beam dynamics is presented. It allows a sraightforward and elegant computation of transfer maps of accelerator systems that can be analysed for data relevant to accelerators. The order of the procedure is unlimited. The theoretical background of the method is presented in detail. Use of the method in practice is shown.

\section{INTRODUCTION}

The effect of an accelerator section can be described mathematically by a map relating the final coordinates $\mathbf{z}_{f}$ of a particle to the initial coordinates $\mathbf{z}_{i}$

$$
z_{f}=M\left(\mathbf{z}_{i}, \boldsymbol{\delta}\right)
$$

The coordinates $\mathbf{z}$ contain positions and momenta of the particle. The vector $\delta$ contains other parameters that influence the motion such as particle energy, mass, or charge or accelerator parameters such as certain multipole strengths. From this map $\mathcal{M}$, quantities of interest for accelerators, such as tune shifts and chromaticities, can be extracted. This is described in detail in a companion paper. ${ }^{1}$

Except for the most trivial cases it is impossible to find a closed analytical solution for the map $\mathcal{M}$. However, expanding $\mathcal{M}$ in a power series yields a set of differential equations for the expansion coefficients that in many cases can be solved analytically up to some order. The complexity of the resulting differential equations, however, increases dramatically with the order of the expansion coefficients. Therefore, this procedure is limited to low or medium orders. In fact, most widely used codes $^{2-4}$ can only compute the nonlinearities in Eq. (1) through third order.

Recently it has been possibe to extend this to higher orders using the custom-made formula manipulator HAMILTON. ${ }^{5}$ This program produces FORTRAN code for formulas of nonlinearities compatible with the program COSY. ${ }^{6}$ At present, COSY can compute all fifth-order nonlinearities of common beam-line elements, such as bending magnets and magnetic and electrostatic multipoles, including the dependence on the particle mass.

Since the accuracy of the Taylor series representation of the map in Eq. (1) is increased by use of higher orders, it is desirable to know $\mathcal{M}$ to as high an order as possible. This certainly holds for the purpose of using the map for 
subsequent symplectic tracking of simulation particles; but also for the abovementioned purely analytical purposes such as computation of tune shifts with amplitude, chomaticities, invariants, etc., based on Hamiltonian perturbation theory, ${ }^{1,7,8}$ this is highly desirable.

In this paper we will present a straightforward way to compute nonlinearities to arbitrary orders based on differential algebraic techniques. The partial derivatives are computed to machine precision; the whole procedure is completely independent of the order and only limited by the power of the computer.

In addition to the elegance with which transfer maps can be produced, the method is so versatile that it allows the computation of arbitrary derivatives. Here no analytic formulas for derivatives must be derived; on the other hand, the method is always accurate to machine precision independent of the order of the derivative, which is in sharp contrast to methods of numerical differentiation.

\section{DIFFERENTIAL ALGEBRAS}

In this section we will provide the mathematical background of the theory of differential algebras required for the promised study of nonlinearities. It is an application of the relatively new field of Nonstandard Analysis, ${ }^{9,10}$ which allows the introduction of arbitrarily small quantities, "infinitesimals," in a rigorous theory of anaiysis. There is also some connection to the theories of formal power series $^{11}$ and automated differentiation. ${ }^{12}$ The use of differential algebras for the field of nonlinear dynamics was first discussed in Ref. 13.

For the sake of clarity, we first address the simplest case of differential algebras, the structure ${ }_{1} D_{1}$.

\subsection{The Structure ${ }_{1} D_{1}$}

Consider the vector space $R^{2}$ of ordered pairs $\left(a_{0}, a_{1}\right), a_{0}, a_{1} \in R$, in which an addition and a scalar multiplication are defined in the usual way:

$$
\begin{aligned}
\left(a_{0}, a_{1}\right)+\left(b_{0}, b_{1}\right) & =\left(a_{0}+b_{0}, a_{1}+b_{1}\right) \\
t \cdot\left(a_{0}, a_{1}\right) & =\left(t \cdot a_{0}, t \cdot a_{1}\right)
\end{aligned}
$$

for $a_{0}, a_{1}, b_{0}, b_{1} \in R$. Besides the above addition and scalar multiplication a multiplication between vectors is introduced in the following way:

$$
\left(a_{0}, a_{1}\right) \cdot\left(b_{0}, b_{1}\right)=\left(a_{0} \cdot b_{0}, a_{0} \cdot b_{1}+a_{1} \cdot b_{0}\right)
$$

for $a_{0}, a_{1}, b_{0}, b_{1} \in R$. With this derinition of a vector multiplication the set of ordered pairs becomes an algebra, denoted by ${ }_{1} D_{1}$.

Note that the multiplication is the same one would obtain by multiplying $\left(a_{0}+a_{1} \cdot x\right)$ and $\left(b_{0}+b_{1} \cdot x\right)$ and keeping terms linear in $x$.

Similarly, as in the case of complex numbers, one can identify $\left(a_{0}, 0\right)$ as the 
real number $a_{0}$. Although as a complex number, $(0,1)$ is a root of -1 , here it has another interesting properı:

$$
(0,1) \cdot(0,1)=(0,0)
$$

which follows directly from Eq. (4). So $(0,1)$ is a root of 0 . Such a property suggests thinking of $d=(0,1)$ as something infinitely small; so small in fact that its square vanishes. Consequently, we call $d=(0,1)$ the differential unit. The first component of the pair $\left(a_{0}, a_{1}\right)$ is called the real part, and the second component is called the differential part.

It is easy to verify that $(1,0)$ is a neutral element of multiplication, because according to Eq. (4)

$$
(1,0) \cdot\left(a_{0}, a_{1}\right)=\left(a_{0}, a_{1}\right) \cdot(1,0)=\left(a_{0}, a_{1}\right) .
$$

It turns out that $\left(a_{0}, a_{1}\right)$ has a multiplicative inverse if and only if $a_{0}$ is nonzero; so, $D_{1}$ is not a field. In case $a_{0} \neq 0$ the inverse is

$$
\left(a_{0}, a_{1}\right)^{-1}=\left(\frac{1}{a_{0}},-\frac{a_{1}}{a_{0}^{2}}\right) .
$$

It is easy to check that in fact $\left(a_{0}, a_{1}\right)^{-1} \cdot\left(a_{0}, a_{1}\right)=(1,0)$.

The space ${ }_{1} D_{1}$ is a subspace of the field $R^{*}$ introduced in Nonstandard Analysis. ${ }^{9,10}$ Besides the usual real numbers, $R^{*}$ contains a variety of infinitely small and infinitely large quantities. The outstanding result of the theory of Nonstandard Analysis is that differentiation becomes an algebraic problem: a function $f$ is differentiable if and only if for any arbitrarily small quantity $\delta$, the real part of the quotient,

$$
\frac{f(x+\delta)-f(x)}{\delta}
$$

is independent of the choice of the specific $\delta$. Thus, given any differentiable function $f$, we can compute its derivatives just by evaluating the formula for a special choice of $\delta$. We choose $\delta=d=(0,1)$ and thus obtain

$$
\begin{aligned}
f^{\prime}(x) & =\mathscr{R}\left[\frac{f(x+d)-f(x)}{d}\right] \text { or } \\
f^{\prime}(x) & =\mathscr{D}[f(x+d)-f(x)]=\mathscr{D}[f(x+d)],
\end{aligned}
$$

where $\mathscr{R}$ denotes the real part, and $\mathscr{D}$ denotes the differential part. In the last step use has been made of the fact that $f(x)$ has no differential part. Hence differential algebras are useful to compute derivatives directly, without requiring an analytic formula for the derivative and without the inaccuracies of numerical techniques.

The computation of derivatives will be illustrated in an example using the following function:

$$
f(x)=\frac{1}{x+\frac{1}{x}}
$$


The derivative of the function is

$$
f^{\prime}(x)=\frac{\frac{1}{x^{2}}-1}{\left(x+\frac{1}{x}\right)^{2}}
$$

Suppose we are interested in the value of the function and its derivative at $x=2$. We obtain

$$
f(2)=\frac{2}{5} \quad f^{\prime}(2)=-\frac{3}{25}
$$

Now if one takes the definition of the function $f$ in Eq. (10) and evaluates it at $2+d=(2,1)$, one obtains

$$
\begin{aligned}
f[(2,1)] & =\frac{1}{(2,1)+\frac{1}{(2,1)}} \\
& =\frac{1}{(2,1)+\left(\frac{1}{2},-\frac{1}{4}\right)} \\
& =\frac{1}{\left(\frac{5}{2}, \frac{3}{4}\right)} \\
& =\left(\frac{2}{5},-\frac{3}{4} / \frac{25}{4}\right) \\
& =\left(\frac{2}{5},-\frac{3}{25}\right) .
\end{aligned}
$$

As we can see, after the evaluation of the function the real part of the result is just the value of the function at $x=2$, whereas the differential part is the derivative of the function at $x=2$.

This is exactly what was to be expected from the theory of Nonstandard Analysis. However, to avoid relying on the quite advanced techniques of this relatively new field of mathematics, we also present an elementary, but less elegant, proof of the result.

By our choice of the starting vector $(2,1)$, initially the vector contains the value $I(2)$ of the identity function $I: x \rightarrow x$ in the first component and the derivative of $I^{\prime}(2)=1$ in the second component.

Now assume that in an intermediate step two vectors of value and derivative $\left[g(2), g^{\prime}(2)\right]$ and $\left[h(2), h^{\prime}(2)\right]$ must be added. According to Eq. (2) one obtains $\left[g(2)+h(2), g^{\prime}(2)+h^{\prime}(2)\right]$. But, according to the rule for the differentiation of sums, this is just the value and derivative of the sum function $(g+h)$ at $x=2$.

The same holds for the multiplication. Suppose thai two ictivis of who and derivatives $\left[g(2), g^{\prime}(2)\right]$ and $\left(h(2), h^{\prime}(2)\right]$ must be multiplied. Then according to Eq. (4) one obtains $\left[g(2) \cdot h(2), g(2) \cdot h^{\prime}(2)+g^{\prime(2)} \cdot h(2)\right]$. But, according to the 
product rule, this is just the value and derivative of the product function $(g \cdot h)$ at $x=2$.

The evaluation of the function $f$ at $(2,1)$ can now be viewed as successively combining two intermediate functions $g$ and $h$, starting with the identity function, and finally arriving at $f$. At each intermediate step the derivative of the intermediate function is automatically obtained as the differential part according (o) the above reasoning.

An interesting sidelight is that, with the search for a multiplicative inverse in Eq. (7), one has derived a rule to differentiate the function $f(x)=1 / x$ without explicitly using calculus rules.

After discussing the algebra ${ }_{1} D_{1}$ and its virtues for the computation of derivatives, we now address a more general differential algebra, the structure ${ }_{n} D_{11}$. It will eventually allow us to compute partial derivatives of functions of $v$ variables through order $n$ arithmetically.

\section{2. The Structure ${ }_{n} D_{v}$}

We define $N(n, v)$ to be the number of monomials in $v$ variables through order $n$. We will show that $N(n, v)=\frac{(n+v) !}{n ! v !}=C(n+v, v)$, where $C(i, j)$ is the familiar binomial coefficient. First note that the number of monomials with exact order $n$ equals $N(n, v-1)$ because each monomial of exact order $n$ can be written as a monomial with one variable less times the last variable to such a power that the total power equals $n$. Thus we have $N(n, v)=N(n-1, v)+N(n, v-1)$ : the number of monomials in $v$ variables through order $n$ equals the number of monomials of one order less plus the ones of exact order $n$. This recursive relation is satisfied by $C(n+v, v)$. Since also, obviously, $C(1+1,1)=2=$ $N(1,1)$, the formula follows by induction.

Now assume that all these $N$ monomials are arranged in a certain manner order by order. For each monomial $M$ we call $I_{M}$ the position of $M$ according to the ordering. Conversely, with $M_{1}$ we denote the $I$ th monomial of the ordering. Finally, for an $I$ with $M_{l}=x_{1}^{i_{1}} \cdots \cdots x_{v}^{i_{y}}$, we define $F_{l}=i_{1} ! \cdot \cdots \cdot i_{v}$ !.

We now define, in addition, a scalar multiplication and a vector multiplication on $R^{N}$ in the following way:

$$
\begin{gathered}
\left(a_{1}, \ldots, a_{N}\right)+\left(b_{1}, \ldots, b_{N}\right)=\left(a_{1}+b_{1}, \ldots, a_{N}+b_{N}\right) \\
t \cdot\left(a_{1}, \ldots, a_{N}\right)=\left(t \cdot a_{1}, \ldots, t \cdot a_{N}\right) \\
\left(a_{1}, \ldots, a_{N}\right) \cdot\left(b_{1}, \ldots, b_{N}\right)=\left(c_{1}, \ldots, c_{N}\right)
\end{gathered}
$$

where the coefficients $c_{i}$ are defined as follows:

$$
c_{i}=F_{i} \sum_{\substack{0 \leq \nu, \mu \leq N \\ M_{v} \cdot M_{\mu}=M_{i}}} \frac{a_{v} \cdot b_{\mu}}{F_{v} \cdot F_{\mu}} .
$$

To help clarify these definitions, let us look at the case of two variables and second order. In this case, we have $n=2$ and $v=2$. There are $N=C(2+2,2)=6$ 
monomials in two variables, namely,

$$
1, x, y, x x, x y, y y \text {. }
$$

As an example, using the ordering in Eq. (18), we have $I_{x y}=5$ and $M_{3}=y$. Using the ordering in Eq. (18), we obtain for $c_{1}$ through $c_{6}$ in Eq. (17):

$$
\begin{aligned}
& c_{1}=a_{1} \cdot b_{1} \\
& c_{2}=a_{1} \cdot b_{2}+a_{2} \cdot b_{1} \\
& c_{3}=a_{1} \cdot b_{3}+a_{3} \cdot b_{1} \\
& c_{4}=2 \cdot\left(a_{1} \cdot b_{4} / 2+a_{2} \cdot b_{2}+a_{4} \cdot b_{1} / 2\right) \\
& c_{5}=a_{1} \cdot b_{5}+a_{2} \cdot b_{3}+a_{3} \cdot b_{2}+a_{5} \cdot b_{1} \\
& c_{6}=2 \cdot\left(a_{1} \cdot b_{6} / 2+a_{3} \cdot b_{3}+a_{6} \cdot b_{1} / 2\right) .
\end{aligned}
$$

On ${ }_{n} D_{v}$ we introduce a third operation $\partial_{i}$ :

with

$$
\partial_{i}\left(a_{1}, \ldots, a_{N}\right)=\left(c_{1}, \ldots, c_{N}\right),
$$

$$
c_{i}=\left\{\begin{array}{ll}
0 & \text { if } M_{i} \text { has order } n \\
a_{I_{\left(M_{i}, x_{1}\right)}} & \text { otherwise }
\end{array} .\right.
$$

So $\partial_{v}$ moves the derivatives around in the vector. Suppose a vector contains the derivatives of the function $f$; then applying $\partial_{v}$ to it one obtains the derivatives of $\frac{\partial f}{\partial x_{v}}$ through one order less. With this third operation, ${ }_{n} D_{v}$, becomes a Differential Algebra as defined in Ref. 14.

Although in , $D_{1}, d=(0,1)$ was an infinitely small quantity, here we have a whole variety of infinitely small quantities with the property that high-enough powers of them vanish. We give special names to the ones in components $I$ belonging to first-order monomials, denoting them by $d M_{l}$. In the example of ${ }_{2} D_{2}$, we have $d x=(0,1,0,0,0,0)$, and $d y=(0,0,1,0,0,0)$. It then follows from the theory of Nonstandard Analysis that instead of $\mathrm{Eq}$. (9) we obtain

$$
f(x+d x, y+d y)=\left(f, \frac{\partial f}{\partial x}, \frac{\partial f}{\partial y}, \frac{\partial^{2} f}{\partial x^{2}}, \frac{\partial^{2} f}{\partial x \partial y}, \frac{\partial^{2} f}{\partial y^{2}}\right)(x, y) .
$$

In the general case of $v$ variables and order $n$, after evaluating $f$ in the differential algebra one obtains

$$
\frac{\partial^{i_{1}+i_{2}+\cdots+i_{1,}} f}{\partial x_{1}^{i_{1}} \partial x_{2}^{i_{2}} \cdots \partial x_{v !}^{i_{1}}}=c_{1_{\left(x_{1}^{\prime}, \cdots \cdot x_{1 ! n}^{\prime}\right)}}
$$

where $I\left(x_{1}^{i_{1}} \cdot \cdots \cdot x_{v}^{i_{1}}\right)$ is the index of the monomial $\left(x_{1}^{i_{1}} \cdots \cdot x_{v}^{i_{v}}\right)$, as defined in the beginning of this section.

\section{IMPORTANT FUNCTIONS IN DIFFERENTIAL ALGEBRAS}

In this section we will generalize standard functions, such as exponentials and logarithmic and trigonometric functions, to differential algebra. As we will see 
helow, virtually all functions existing on a computer can be generalized straightforwardly.

We start our discussion by noting that for any differential-algebra vector of the form $\left(0, a_{1}, \ldots, a_{N}\right) \in_{n} D_{v}$, i.e. with a zero in the component belonging to the croth-order monomial, we have the following property:

$$
\left(0, a_{1}, \ldots, a_{N}\right)^{i}=(0,0, \ldots, 0) \text { for } i>n,
$$

which follows directly from the definition of the multiplication in ${ }_{n} D_{v}$ defined in Eq. (16).

Let us begin our discussion of special functions with the exponential function $\exp (x)$. Assume we have to compute the exponential of a differential-algebra vector that has already been created by previous operations. First we note that the functional equation $\exp (x+y)=\exp (x) \cdot \exp (y)$ also holds in Nonstandard Analysis. As we will see, this facilitates considerably the computation of the exponential. We obtain

$$
\begin{aligned}
\exp \left[\left(a_{0}, a_{1}, a_{2}, \ldots, a_{N}\right)\right] & =\exp \left(a_{0}\right) \cdot \exp \left[\left(0, a_{1}, a_{2}, \ldots, a_{N}\right)\right] \\
& =\exp \left(a_{0}\right) \cdot \sum_{i=0}^{\infty} \frac{\left(0, a_{1}, a_{2}, \ldots, a_{N}\right)^{i}}{i !} \\
& =\exp \left(a_{0}\right) \cdot \sum_{i=0}^{n} \frac{\left(0, a_{1}, a_{2}, \ldots, a_{N}\right)^{i}}{i !}
\end{aligned}
$$

In the last step Eq. (24) was used. This entails that the sum has to be taken through only order $n$, which thus allows the computation of the exponential in a finite number of steps. Hence the evaluation of the real number exponential $\exp \left(a_{1}\right)$, which internally on a computer requires a power series summation and hence cannot be done accurately, is more subtle than the rest of the operations in differential algebra.

A logarithm of a differential-algebra vector exists if and only if $a_{0}>0$. In this case one obtains

$$
\begin{aligned}
\log \left[\left(a_{0}, a_{1}, a_{2}, \ldots, a_{N}\right)\right] & =\log \left\{a_{0} \cdot\left[1+\left(0, \frac{a_{1}}{a_{0}}, \frac{a_{2}}{a_{0}}, \ldots, \frac{a_{N}}{a_{0}}\right)\right]\right\} \\
& =\left[\log \left(a_{0}\right), 0, \ldots, 0\right]+\sum_{i=1}^{\infty}(-1)^{i+1} \frac{1}{i}\left(0, \frac{a_{1}}{a_{0}}, \frac{a_{2}}{a_{0}}, \ldots, \frac{a_{N}}{a_{0}}\right)^{i} \\
& =\left[\log \left(a_{0}\right), 0, \ldots, 0\right]+\sum_{i=1}^{n}(-1)^{i+1} \frac{1}{i}\left(0, \frac{a_{1}}{a_{0}}, \frac{a_{2}}{a_{0}}, \ldots, \frac{a_{N}}{a_{0}}\right)^{i}
\end{aligned}
$$

Again use has been made of the fundamental property of the logarithm $\log (x \cdot y)=\log x+\log y$, which transforms directly into Nonstandard Analysis and leads to simplifications by virtue of Eq. (24).

As the last example, we will derive a formula for the root function. Even though there is a direct method to compute roots by solving a set of linear equations for the coefficients of the root, here we present a technique based on power series following an approach similar to the exponential and logarithm. The 
root has the following power-series expansion:

$$
\sqrt{1+x}=\sum_{i=0}^{\infty}(-1)^{i} \frac{1 \cdot 3 \cdot \cdots \cdot(2 i-3)}{2 \cdot 4 \cdot \cdots \cdot(2 i)} \cdot x^{i} .
$$

Using this formula and the definitions of addition and multiplication in Eqs. (14) and (16), one directly obtains for the square root of a differential-algebra vector:

$$
\begin{aligned}
& \sqrt{\left(a_{0}, a_{1}, a_{2}, \ldots, a_{N}\right)} \\
= & \sqrt{a_{0}} \cdot \sqrt{1+\left(0, \frac{a_{1}}{a_{0}}, \frac{a_{2}}{a_{0}}, \ldots, \frac{a_{N}}{a_{0}}\right)} \\
= & \sqrt{a_{0}} \cdot \sum_{i=0}^{\infty}(-1)^{i} \frac{1 \cdot 3 \cdot \cdots \cdot(2 i-3)}{2 \cdot 4 \cdot \cdots \cdot(2 i)} \cdot\left(0, \frac{a_{1}}{a_{0}}, \frac{a_{2}}{a_{0}}, \frac{a_{N}}{a_{0}}\right)^{i} \\
= & \sqrt{a_{0}} \cdot \sum_{i=0}^{n}(-1)^{i} \frac{1 \cdot 3 \cdot \cdots \cdot(2 i-3)}{2 \cdot 4 \cdot \cdots \cdot(2 i)} \cdot\left(0, \frac{a_{1}}{a_{0}}, \frac{a_{2}}{a_{0}}, \ldots, \frac{a_{N}}{a_{0}}\right)^{i} .
\end{aligned}
$$

Using the addition theorems for sine and cosine, one obtains formulas with finite sums in a quite-similar way; in general, suppose a function $f$ has an addition theorem of the form

$$
f(a+b)=g_{a}(b)
$$

and $g_{a}(b)$ can be written in a power series, then by the same reasoning its differential-algebraic extension can be computed exactly in a finite number of steps. In practice it turns out that this can be done for all commonly supported functions in a FORTRAN computer environment.

\section{THE IMPLEMENTATION OF DIFFERENTIAL ALGEBRA ON A COMPUTER}

The arithmetic and the functions of differential algebra can be implemented on a computer for arbitrary order and an arbitrary number of variables. As it turns out, this is not easily done for the field $R^{*}$ of Nonstandard Analysis; hence we sacrifice the universal existence of multiplicative inverses.

The implementation of the addition and scalar multiplication is trivial. However, the efficient implementation of a multiplication requires some care. First we note that we can increase the speed by defining a multiplication that differs slightly from that in Eq. (17), with the $c_{i}$ as

$$
c_{i}=\sum_{\substack{0<v, \mu=N \\ M_{v} \cdot M_{\mu}=M_{i}}} a_{v} \cdot b_{\mu} .
$$

This multiplication is the same as in the case of the multiplication of power series. However, in this arithmetic not all power-series coefficients of the product nes romputed; conffiniontc holnoning to terms of orders higher than $n$ are disregarded. For many cases this view of differential algebras as "truncated power-series algebras" is sufficient (see Ref. 13). 
The definition of the multiplication in Eq. (16) requires the knowledge of all pessible factorizations of a monomial into two submonomials. The computation of all these factorizations can be quite time consuming. Additionally, in practice it happens frequently that many of the entries in a differential-algebra vector are acro.

So it is advantageous to turn the problem inside out so that no factorizations in submonimials are searched, but rather each component of the first vector is multiplied by each component of the second vector, and the product is stored where the product monomial belongs.

To do that requires an easy way of finding the address of the product monomial. This is done as follows. First, all $N(n, v)$ monomials $M$ are coded with an integer $C$ in the following way: let $M=x_{1}^{i_{1}} \cdot \cdots \cdot x_{v}^{i_{1}}$. Then we define $C(M)$ as follows:

$C(M)=C\left(x_{1}^{i_{1}} \cdot \cdots \cdot x_{v}^{i_{v}}\right)=i_{1} \cdot(n+1)^{0}+i_{2} \cdot(n+1)^{1}+\cdots+i_{v} \cdot(n+1)^{(v-1)}$.

This means that the exponents are just "decimals" in base $(n+1)$. Note that since $i_{v} \leq n$ this representation is injective; i.e., different monomials have different codings. Note also that all codings are always less than $(n+1)^{v}$, but not all such codings occur.

Now suppose two monomiais $M$ and $N$ must be multiplied and suppose their product has an order less than or equal to $v$. Since the multiplication corresponds (o) an addition of the exponents, it follows that

$$
C(M \cdot N)=C(M)+C(N) .
$$

To exploit this to find the desired coordinate position $I_{M}$ (see Section 2) of the product of two monomials, an array $D$ is required that has the property

$$
I_{M}=D[C(M)]
$$

This array can be generated easily by the computer. Since the codings are bounded by $(n+1)^{v}$, the array has to have at least this length. With six variables, this enables orders of eight or nine if one wants to stay inside the boundaries of computer storage; with eight variables the order would decrease to about four, which is too strict a limitation. To circumvent this, a slight modification of the above coding and decoding, will be presented.

Without loss of generality, we assume the number of variables $v$ to be even; if it is not even, increase it by one, and ignore the additional variable. We define two coding numbers $C_{1}$ and $C_{2}$ for any monomial in the following way:

$$
\begin{aligned}
C_{1}\left(x_{1}^{i_{1}} \cdot \ldots \cdot x_{v}^{i_{y}}\right) & =i_{1} \cdot(n+1)^{0}+i_{2} \cdot(n+1)^{1}+\cdots+i_{\frac{v}{2}} \cdot(n+1)^{\left(\frac{1}{2}-1\right)} \\
C_{2}\left(x_{1}^{i_{1}} \cdot \ldots \cdot x_{v}^{i_{v}}\right) & =i_{\frac{v}{2}+1} \cdot(n+1)^{0}+i_{\frac{v}{2}+2} \cdot(n+1)^{1}+\cdots+i_{v} \cdot(n+1)^{\left(\frac{v}{2}-1\right)} .
\end{aligned}
$$

Then we store the $N(n, v)$ monomials in the following way. We start with all monomials that have $C_{2}(M)=0$ and group them by order; within one order, the monomials are stored according to ascending values of $C_{1}(M)$. Then we store all those with $C_{2}(M)=1$, again by order, and so forth, going through all possible 
values of $C_{2}$. Again we obtain

$$
\begin{aligned}
& C_{1}(M \cdot N)=C_{1}(M)+C_{1}(N) \\
& C_{2}(M \cdot N)=C_{2}(M)+C_{2}(N) .
\end{aligned}
$$

Finally we introduce some "inverse" arrays $D_{1}$ and $D_{2}$ in the following way:

$$
\begin{aligned}
& D_{1}\left(c_{1}\right)=\left(I_{M} \text { of first monomial } M \text { with } C_{1}(M)=c_{1}\right) \\
& D_{2}\left(c_{2}\right)=\left(I_{M} \text { of first monomial } M \text { with } C_{2}(M)=c_{2}\right)-1
\end{aligned}
$$

Again the arrays $D_{1}$ and $D_{2}$ can be generated by the computer.

\section{TABLE I}

\begin{tabular}{|c|c|c|c|c|c|c|}
\hline$I_{M}$ & $i_{1}$ & $i_{2}$ & $i_{3}$ & $i_{4}$ & $C_{1}$ & $c_{2}$ \\
\hline 1 & 0 & 0 & 0 & () & () & 0 \\
\hline 2 & 1 & 0 & 0 & 0 & 1 & 0 \\
\hline 3 & 0 & 1 & () & 0 & 4 & () \\
\hline 4 & 2 & 0 & 0 & 0 & 2 & 0 \\
\hline 5 & 1 & 1 & 0 & () & 5 & () \\
\hline 6 & 0 & 2 & 0 & 0 & 8 & 0 \\
\hline 7 & 3 & 0 & 0 & 0 & 3 & () \\
\hline 8 & 2 & 1 & 0 & 0 & 6 & 0 \\
\hline 9 & 1 & 2 & 0 & 0 & 9 & 0 \\
\hline 10 & 0 & 3 & 0 & 0 & 12 & 0 \\
\hline 11 & 0 & 0 & 1 & 0 & 0 & 1 \\
\hline 12 & 1 & 0 & 1 & 0 & 1 & 1 \\
\hline 13 & 0 & 1 & 1 & () & 4 & 1 \\
\hline 14 & 2 & 0 & 1 & 0 & 2 & 1 \\
\hline 15 & 1 & 1 & 1 & 0 & 5 & 1 \\
\hline 16 & 0 & 2 & 1 & 0 & 8 & 1 \\
\hline 17 & 0 & 0 & 0 & 1 & () & 4 \\
\hline 18 & 1 & 0 & 0 & 1 & 1 & 4 \\
\hline 19 & 0 & 1 & 0 & 1 & 4 & 4 \\
\hline 20 & 2 & 0 & 0 & 1 & 2 & 4 \\
\hline 21 & 1 & 1 & 0 & 1 & 5 & 4 \\
\hline 22 & 0 & 2 & 0 & 1 & 8 & 4 \\
\hline 23 & 0 & 0 & 2 & 0 & 0 & 2 \\
\hline 24 & 1 & 0 & 2 & 0 & 1 & 2 \\
\hline 25 & 0 & 1 & 2 & 0 & 4 & 2 \\
\hline 26 & 0 & 0 & 1 & 1 & () & 5 \\
\hline 27 & 1 & 0 & 1 & 1 & 1 & 5 \\
\hline 28 & () & 1 & 1 & 1 & 4 & 5 \\
\hline 29 & 0 & 0 & 0 & 2 & () & 8 \\
\hline 30 & 1 & 0 & 0 & 2 & 1 & 8 \\
\hline 31 & () & 1 & 0 & 2 & 4 & 8 \\
\hline 32 & 0 & 0 & 3 & 0 & 0 & 3 \\
\hline 33 & 0 & 0 & 2 & 1 & 0 & 6 \\
\hline 34 & 0 & 0 & 1 & 2 & 0 & 9 \\
\hline 35 & 0 & 0 & 0 & 3 & 0 & 12 \\
\hline
\end{tabular}

List of the ordering of all the monomials $M=x_{1}^{i_{1}} \cdot \cdots \cdot x_{v}^{i_{1}}$ for order $n=3$ and number of variables $v=4$ and the coding integers $C_{1}$ and $C_{2}$ 
With the definitions of $C_{1}, C_{2}, D_{1}$, and $D_{2}$ and the storage scheme outlined above, it now follows that the address of the product of the monomials $M$ and $N$ cin be found directly as

$$
I_{M \cdot N}=D_{1}\left[C_{1}\left(I_{M}\right)+C_{1}\left(I_{N}\right)\right]+D_{2}\left[C_{2}\left(I_{M}\right)+C_{2}\left(I_{N}\right)\right] .
$$

For the sake of clarity, examples for the arrays $c_{1}, c_{2}, d_{1}$, and $d_{2}$ are given in Tables I and II for $n=3$ and $v=4$. These examples also illustrate Eqs. (34) through (37).

\section{TABLE II}

List of the arrays $D_{1}$ and $D_{2}$ for order $n=3$ and number of variables $v=4$

\begin{tabular}{rcc}
\hline$j$ & $D_{1}(j)$ & $D_{2}(j)$ \\
\hline 0 & 1 & 0 \\
1 & 2 & 10 \\
2 & 4 & 22 \\
3 & 7 & 31 \\
4 & 3 & 16 \\
5 & 5 & 25 \\
6 & 8 & 32 \\
7 & 0 & 0 \\
8 & 6 & 28 \\
9 & 9 & 33 \\
10 & 0 & 0 \\
11 & 0 & 0 \\
12 & 10 & 34 \\
\hline
\end{tabular}

For all $M$, one has $I_{M}=D_{1}\left[C_{1}(M)\right]+$ $D_{2}\left[C_{2}(M)\right]$

The coding defined in Eq. (34) allows the maximum length of the arrays $D_{1}$ and $D_{2}$ to be chosen much lower, namely as $(n+1)^{\frac{v}{2}}$.If a maximum length of 1 million is assumed, this entails the limitations on the maximum order given a certain number of variables that are listed in Table III.

After addition and multiplication are available, the implementation of

\section{TABLE III}

Maximum order for different numbers of variables due to a limitation on the length of the reverse addressing arrays $D_{1}, D_{2}$

\begin{tabular}{cc}
$\begin{array}{c}\text { Number of } \\
\text { variables }\end{array}$ & $\begin{array}{c}\text { Maximum } \\
\text { order }\end{array}$ \\
\hline 6 & 99 \\
8 & 30 \\
10 & 14 \\
12 & 10 \\
\hline
\end{tabular}


differential-algebra functions is done quite easily using the fomulas discussed in Section 3.

For practical purposes it is of importance that in the FORTRAN environment differential-algebraic operations can only be used by calls to subroutines. For this reason a precompiler ${ }^{15}$ was developed that allows the use of a new data type "differential algebra" in regular FORTRAN formulas. The precompiler parses the entire program and transforms formulas containing differential-algebraic quantities into subroutine calls.

\section{THE COMPUTATION OF TRANSFER MAPS}

\subsection{An Illustrating Example}

Differential Algebras can be used quite efficiently to compute the transfer map of Eq. (1) of particle optical systems in its Taylor series representation.

To illustrate this, let us start the discussion with a simple example, the midplance motion in a $90^{\circ}$ homogenous bending magnet. Let $x_{i}$ and $a_{i}=\sin \alpha_{i}$ denote the initial distance and scaled transverse momentum relative to the reference trajectory (see Fig. 1). Then we are interested in the values $x_{f}$ and $a_{f}=\sin \alpha_{f}$. Since the trajectories in the magnet are circles, we can readily read from Fig. 1:

$$
\begin{aligned}
& A=R \sin \alpha_{i}=R a_{i} \\
& B=R\left(1-\cos \alpha_{i}\right)+x_{i}=R\left(1-\sqrt{1-a_{i}^{2}}\right)+x_{i} \\
& a_{f}=\sin \alpha_{f}=-\frac{B}{R} \\
& x_{f}=A-R\left(1-\cos \alpha_{f}\right)=A-R\left(1-\sqrt{1-a_{f}^{2}}\right) .
\end{aligned}
$$

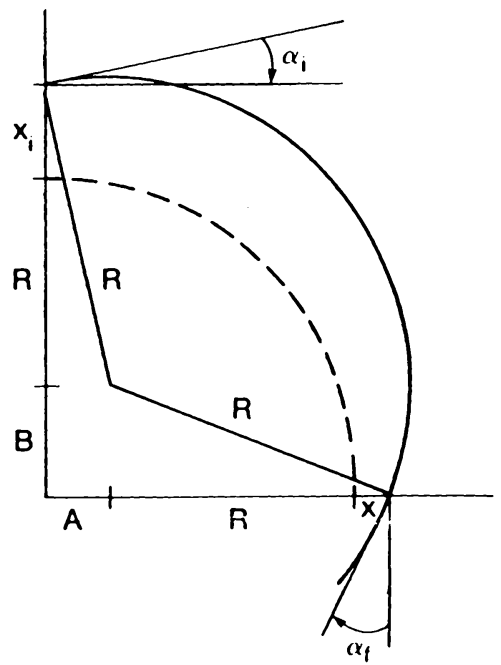

FIGURE 1 
These cquations allow the computation of the final coordinates $x_{f}, a_{f}$ in terms of the initial coordinates $x_{i}, a_{i}$. However, taking these equations and performing all operations in differential algebra even allows us to obtain all derivatives of $x_{f}$, a, with respect to $x_{i}, a_{i}$. These so-obtained derivatives, evaluated at $x_{i}=0, a_{i}=0$, are then the expansion coefficients of the map of Eq. (1). For the sake of clarity, let us explicitly show how $x_{f}$ and $a_{f}$ are computed.

Using the ordering in Eq. (18) and identifying the variable $a$ with $y$, we obtain, using the arithmetic defined in Eqs. (14), (15), and (16),

$$
\begin{aligned}
x_{i} & =(0,1,0,0,0,0) \\
a_{i} & =(0,0,1,0,0,0) \\
A & =(0,0, R, 0,0,0,) \\
B & =(0,1,0,0,0, R) \\
a_{f} & =\left(0,-\frac{1}{R}, 0,0,0,-1\right) \\
x_{f} & =\left(0,0, R,-\frac{1}{R}, 0,0\right) .
\end{aligned}
$$

Comparing the so-obtained result with any matrix code, ${ }^{24.6}$ we find complete agreement; as an example, the fact that the second component of $x_{f}$ is zero implies that $\frac{\partial x_{f}}{\partial x_{i}}=0$ and hence $(x, x)=0$ (or in TRANSPORT notation $R_{1,1}=0$ ), which is a well-known property of $90^{\circ}$ bends.

In case an additional particle optical element is to follow this bending magnet, one need not begin evaluating this new element at $x_{i}=(0,1,0,0,0,0)$, $a_{1}=(0,0,1,0,0,0)$, but one can start with $x_{f}$ and $a_{f}$ of Eq. (39). This way one can eliminate the usually quite involved concatenation process and increase performance significantly.

The example discussed in this section has been implemented on the computer. Using the Differential Algebra package, one can easily extract all nonlinearities of this two-dimensional example through order 50 .

\subsection{Generation of Maps Using Numerical Integration}

In this section we will address the general case in which no closed solution of the problem exists. We will see that even in this case we are actually able to compute transfer maps of arbitrary order for arbitrary particle optical elements. Even though we do not have analytical formulas that relate the final coordinates to the initial coordinates, there is still a way to computationally relate the final coordinates to the initial coordinates, by numerical integration of the equations of motion.

In this case, the final coordinates are still computed from the initial coordinates using standard arithmetic and functions; however, the relations are more complex than in the case of the homogeneous sector.

Now blindly performing all these operations in differential algebra automati- 
cally leads to all desired derivatives of the transfer function, regardless of the form of the equations of motion.

Differential-algebraic techniques have been implemented in the program COSY. ${ }^{6}$ They allow the computation of transfer maps of elements, such as fringing fields, with a dependence on the independent variable for which an analytic solution cannot be obtained from HAMILTON. ${ }^{5}$ By use of an eighthorder Runge Kutta integrator, all operations required for a tracking of particles are performed in differential algebra. This allows the computation of arbitrary fringing-field effects as soon as the spatial distribution of the electromagnetic fields is known. ${ }^{16}$

In many cases, including the proposed $\mathrm{SSC},{ }^{17}$ the particle optical system can be represented very well by a sequence of kicks, as in TEAPOT. ${ }^{18}$ The sequence of kicks can be viewed as a symplectic integrator of second order in the independent variable. In this case, the execution of all arithmetic in differential algebra is particularly easy. The so-obtained nonlinear map may be used for very efficient and accurate computation of tune shifts and chromaticities using normal form theory. ${ }^{7,8}$ This is discussed in detail in Ref. 1 .

It is worth mentioning that the maps obtained from TEAPOT and COSY are in complete agreement for all nonlinearities through fifth order. To show this, we selected a small ring for which bends and quadrupoles of finite length had to be split into several hundred drifts and kicks to be computable by TEAPOT.

\subsection{Hamiltonian Theory}

In this subsection we will outline the usefulness of differential algebras for Hamiltonian systems. One of the most fundamental concepts of Hamiltonian theory is the Poisson bracket between two functions of phase space. This requires the differentiation with respect to phase-space variables.

Suppose a differential-algebra vector is given. Then it can be viewed as a descriptor of a function, giving its value and derivatives at a certain point. In this context the required differentiation is just the "bookkeeping operation," moving derivatives in the vector to different places introduced in Section 2.2. Thus a Poisson bracket for arbitrary order and an arbitrary number of variables can be computed.

Using the Poisson bracket, Lie operators, "Poisson brackets waiting to happen," can be computed. In fact, as soon as the generator $\mathbf{v}$ of the Lie operator $: \vec{v}$ : vanishes at the origin and has zero first derivatives, the process is closed in that no feeddown from higher order occurs.

Using Lie operators, the transfer map or flow of a time-independent Hamiltonian system can be computed as

$$
\mathcal{M}=\exp (-t: H:),
$$

where $H$ is the Hamiltonian of the system. Note that by the proper choice of the coordinates it can always be achieved that $H(\overrightarrow{0})=0$ and also the first derivatives of $H$ vanish. This entails that each summand in Eq. (40) can be computed in a closed fashion. 
Furthermore, it turns out that it suffices to compute only a finite number of tcrms of the sum in Eq. (40) if we want the nth-order coefficients of the transfer map to a certain accuracy. To see this, we show that the norm of the Lie operator $:-t H$ : is bounded. First note that to obtain the transfer map through order $n$, it suffices to know $H$ to order $n+1$.

We define a norm on Differential-Algebra vectors in the following way:

$$
\left|\left(a_{1}, \ldots, a_{N}\right)\right|=\sum_{i=1}^{N}\left|a_{i}\right| .
$$

It is quite easy to show that for arbitrary vectors $V_{1}, V_{2}$ we have $\left|V_{1} \cdot V_{2}\right| \leq$ $n \cdot|V| \cdot\left|V_{2}\right|$. Since the "derivative" $\partial_{v}$ defined in Section 2.2 only moves coefficients around in the vector and drops some, it follows for a vector $V$ that

$$
\left|\partial_{v} V\right| \leq|V| \text {. }
$$

Thus we obtain for the norm of the Lie operator consisting of the two derivatives in each summand:

$$
\begin{aligned}
|:-t H:| & =\sup \frac{|-t: H: V|}{|V|} \\
& =\sup \frac{\sum_{i=1}^{v / 2}|t| \cdot\left[\left(\partial_{i} H\right)\left(\partial_{i+v / 2} V\right)-\left(\partial_{i} V\right)\left(\partial_{i+v / 2} H\right)\right]}{|V|} \\
& \leq|t| \cdot|H| \cdot v .
\end{aligned}
$$

This entails that the sum $\sum_{v=0}^{\infty} \frac{(-t: H:)}{v !}$ converges (in a differential-algebra sense!), and that it is possible to estimate how many terms must be taken to obtain a certain accuracy. In general it turns out that these estimates are usually quite pessimisitc, and the terms in the sum become small more quickly.

This method to obtain the Hamiltonian flow in a computer code has the additional advantage that it does not require any composition of the maps of two subsystems; all that is required to compute $H$ by substituting in the $z$ already obtained and then let this differential-algebraic vector act on $\mathbf{z}$.

In practice it turns out that the savings from the avoided compositions are more significant than the additional time required for the iterative evaluation of the exponential sum $\exp (:-t H:)$. In fact, a composition routine requires $N(n, v)$ differential-algebra multiplications, whereas one term in the sum requires $v$ multiplications per dimension.

In the case where the Hamiltonian is time dependent, the above reasoning requires a slight modification. In this case it is, in general, not possible to go through the time-dependent element in one step. However, it is possible to derive an explicit high-order numerical integrator in the following way. First we note that for any function of phase space, we have

$$
\frac{d f}{d t}=(f, H)+\frac{\partial}{\partial t} f=O f .
$$

In our particular case, we are especially interested in the cases in which $f$ are the components of $\mathbf{z}$. Iterating Eq. (44), we can obtain higher-order derivatives of 
$f$, say up to order $u$. First we note that even for the $f$ 's of interest, $\frac{\partial}{\partial t} f$ vanishes, but in the higher derivatives such partial derivatives prevail because $H$ is time dependent.

To guarantee that all derivatives of the phase-space variables can be computed to order $u, H$ must be known to order $v+u$. However, terms with a power of $t$ higher than $u$ can be set to zero. To compute the $u$ derivatives of the phase-space vector, which is needed for the $u$ th-order integrator, a total of $6 \cdot u$ Poisson brackets are required. Considering that in many practical cases an element can be transversed in one step, or very few steps, of an eigth-order integrator and that, as described above, we still save the composition process, the effort is still very favorable to the situations in which compositions are required.

\section{ACKNOWLEDGMENTS}

For financial support I want to thank the Deutsche Forschungsgemeinschaft, Dr. Alex Chao of the SSC Central Design Group, and Dr. Edward Heighway of Los Alamos National Laboratory. For fruitful discussions I want to thank Dr. Etienne Forest and Professor Dr. Hermann Wollnik.

\section{REFERENCES}

1. E. Forest, M. Berz, and J. Irwin, Particle Accelerators 24, 91 (1989).

2. K. L. Brown, D. C. Carey, Ch. Iselin, and F. Rothacker, SLAC 91 (1973 rev.), NAL 91, and CERN 80-84.

3. A. J. Dragt et al., IEEE Trans. Nucl. Sci. NS-32, 2311 (1985).

4. H. Wollnik, J. Brezina, M. Berz, and W. Wendel, Proc. AMCO-7, (GSI-Rep., THD-26, 1984) p. 679.

5. M. Berz and H. Wollnik, Nucl. Inst. Meth. A258, 364 (1987).

6. M. Berz, H. Wollnik, and H. C. Hofmann, Nucl. Inst. Meth. A258, 402 (1987).

7. E. Forest, Particle Acclerators 22, 15 (1987).

8. E. Forest, SSC Report SSC-111, Berkeley (1986)

9. A. Robinson, Proc. Royal Acad. Amsterdam Ser. A64, 432-440, (1961).

10. D. Laugwitz, J. Ber. Deutsch. Math. Vereinigung 75, 66 (1973).

11. I. Niven, Am. Math. Monthly 76-8, 871 (1969).

12. L. B. Rall, Math. Magazine 59, 275 (1986).

13. M. Berz, Nucl. Inst. Meth. A258, 431 (1987); M. Berz, Los Alamos Accelerator Theory Note, AT-6:ATN-86-16 (1986).

14. J. F. Ritt, Differential Algebra, American Mathematical Socicty, 1950.

15. M. Berz, Los Alamos Report AT-3:TN-87-32 (1987).

16. M. Berz, Dissertation University of Giessen, 1986 (unpublished, in German); B. Hartmann, Diplomarbeit University of Giessen 1987 (unpublished, in German).

17. SSC Conceptual Design Report, SSC Note SSC-SR 2020, Berkeley.

18. L. Schachinger and R. Talman, Particle Accelerators 22, 35 (1987). 